INRA Prod. Anim.,

2014, 27 (4), 299-302

\title{
Phénotypage et génotypage de la composition fine du lait : les filières laitières et la recherche française investissent pour l'avenir
}

\author{
M. BROCHARD ${ }^{1,2,3}$, K. DUHEM ${ }^{1}$, T. GESLAIN ${ }^{4}$, P.-L. GASTINEL ${ }^{1}$, J.-L. PEYRAUD ${ }^{5,6}$ \\ ${ }^{1}$ Institut de l'Élevage, 149 rue de Bercy, F-75012 Paris, France \\ 2 INRA, UMR1313 GABI, F-78352 Jouy-en-Josas, France \\ 3 AgroParisTech, UMR1313 GABI, 16 rue Claude Bernard, F-75321 Paris, France \\ ${ }^{4}$ Cniel, 42 rue Châteaudun, F-75009 Paris, France \\ 5 INRA, UMR1348 PEGASE, F-35590 Saint Gilles, France \\ ${ }^{6}$ Agrocampus Ouest, UMR1348 PEGASE, F-35000 Rennes, France \\ Courriel : mickael.brochard@idele.fr
}

\begin{abstract}
PhénoFinlait a été un programme d'envergure ambitieux dont les résultats, les nouvelles références, les échantillons et les bases de données, constituent un solide investissement pour l'avenir des filières laitières françaises.
\end{abstract}

Le programme PhénoFinlait, s'est intéressé à la composition des laits en acides gras et protéines en considérant les trois espèces de ruminants laitiers utilisées en France et est l'un des tous premiers programme de phénotypage animal à haut débit réalisé en France. C'était pour le moins un programme ambitieux par son ampleur, par le nombre de défis scientifiques, techniques et organisationnels qui étaient à relever, sans oublier le défi financier (le budget global du programme était de plus de $4 \mathrm{M} €$ ). Les objectifs initiaux ont été atteints permettant, dès à présent et pour quelques années encore, des exploitations des résultats scientifiques et techniques nombreuses et riches. Les facteurs de réussite principaux ont été la motivation forte et la synergie de l'ensemble des acteurs et métiers fédérés au sein du consortium; le recours à des réseaux préexistants très bien organisés et ayant une grande habitude de travail avec les équipes nationales chargées du projet; un montage et une conduite en parfaite pluridisciplinarité permettant d'anticiper les questions et les potentiels points de difficulté ; et un intérêt certain des éleveurs pour ce sujet, probablement au-delà de leur métier d'éleveur. Nous nous proposons ici de résumer brièvement les principaux acquis du programme, développés dans les articles de ce dossier - Gelé et al (2014), Legarto et al (2014) et Boichard et al (2014) - et d'en dresser les principales perspectives.

\section{1 / Les principaux résultats du programme PhénoFinlait}

Les travaux de PhénoFinlait montrent que l'on peut estimer avec une bonne fiabilité (ajustement supérieur à $80 \%$ et erreur d'estimation relative inférieure à $10 \%$ ) une vingtaine d'acides gras majeurs (Ferrand-Calmels et al 2014), mais aussi plusieurs lactoprotéines majeures. Plus encore, l'application en conditions « réelles » à grande échelle (près de 870000 analyses de lait ont été réalisées) de cette méthodologie s'est révélée tout à fait pertinente comme le montrent les résultats de l'effet de l'alimentation sur la composition fine des laits obtenus par Legarto et al (2014, ce numéro) et les résultats relatifs au déterminisme génétique de ces caractères obtenus par Boichard et al (2014, ce numéro) à partir de ces estimations. Concernant les protéines, il faut ajouter le développement d'une nouvelle méthode d'ancrage particulièrement résolutive, s'appuyant sur une chromatographie en phase liquide couplée à une spectrométrie de masse (Miranda et al 2011). Cette méthode permet d'identifier et quantifier une très grande diversité de protéines et de variants protéiques (génétiques, modifications post-traductionnelles), ainsi que certains produits de la protéolyse.

Les travaux de PhénoFinlait ont confirmé et précisé, en conditions d'élevages commerciaux et à très grande échelle, que plusieurs leviers sont disponibles pour adapter la composition du lait aux trois niveaux de l'animal, du troupeau et du bassin de collecte.

Les teneurs relatives en acides gras des laits peuvent être modulées par l'alimentation (augmentation des acides gras monoinsaturés et diminution $\mathrm{du}$ C16:0 avec les régimes à base d'herbe pâturée par rapport aux régimes à base d'ensilage de maïs). Le programme PhénoFinlait a également permis de préciser l'influence de la race, de facteurs physiologiques (stade de lactation...) ou encore de la saison (après correction pour les facteurs d'alimentation et physiologiques). Par ailleurs, les teneurs en acides gras sont héritables (héritabilités comprises entre 0,10 et 0,40 selon les acides gras). Concernant les variations des teneurs en protéines, il apparaît, comme attendu, que le facteur génétique est beaucoup plus déterminant que le facteur élevage (héritabilité comprise selon les protéines et les espèces entre 0,20 et 0,60 pour les teneurs par contrôle et atteignant jusqu'à 0,82 pour les teneurs moyennes par lactation).

Enfin, de nombreux QTL (« Quantitative Trait Loci ») ont été détectés. Une partie d'entre eux co-localisent avec des gènes connus (DGAT1, SCD, AGPAT6, GHR...). De même, une part importante des QTL détectés co-localisent entre races $(50 \%$ des QTL dans l'espèce bovine) ce qui est plutôt favorable à la compréhension des mécanismes, ainsi qu'à la mise en œuvre d'une sélection. 
Il y a bon espoir de pouvoir identifier quelques nouvelles mutations causales, mais d'ores et déjà ce travail a permis de définir les potentialités de sélection ainsi que les liaisons génétiques à considérer.

\section{2 / Le programme Phéno- Finlait ouvre de nouvelles perspectives}

Le programme PhénoFinlait a permis des progrès décisifs dans l'utilisation de la spectrométrie dans le Moyen-InfraRouge (MIR) pour l'analyse des laits. L'une des toutes premières utilisations de ces avancées a été la qualification physicochimique à grande échelle des laits avec la réalisation d'un état des lieux en fonction des systèmes d'élevage, races et espèces laitières, en France. Sur la base de ces éléments et en s'appuyant sur des méthodes plus résolutives on peut envisager des dispositifs plus restreints, mais judicieusement ciblés d'analyse de la composition " ultra-fine » du lait. Ainsi, concernant les protéines, la méthode développée par Miranda et al (2011) peut être mise en œuvre pour détecter de nouveaux variants protéiques spécifiques de races ou bien encore pour suivre des variants d'intérêt particulier. Un processus de normalisation est d'ores et déjà engagé auprès de la Fédération Internationale Laitière.

Le dispositif PhénoFinlait a permis de préciser au sein de différents bassins de production, espèces et races la variabilité « réellement disponible » et son origine (alimentation, statut physiologique des animaux, génome). Nous n'en sommes encore qu'au début, mais il est vraisemblable que sur la base des ces références, des outils de suivi et d'anticipation de la composition du lait se déploieront afin de mieux piloter la qualité du lait. Le programme PhénoFinlait développe ainsi un outil à l'échelle de l'élevage permettant de comprendre la composition du lait en différents acides gras lors du passage des contrôleurs laitiers et de prédire, en fonction d'un scénario alimentaire et de l'évolution du cheptel en production, la composition du lait au contrôle laitier suivant. Ce type de démarche pourra être appliquée à d'autres composants en fonction de la précision et de la fiabilité des équations d'estimation correspondantes (autres acides gras, protéines majeures), et à d'autres échelles (animal, bassin de collecte, région ou pays). Le déploiement des principales équations d'estimation du profil en acides gras dans les laboratoires d'analyse du lait est désormais une réalité grâce à des accords avec les constructeurs Foss et Bentley Instrument. Les conditions techniques sont également réunies pour enregistrer en routine les données spectrales MIR afin de permettre de nouveaux développements (équations non déployées, en cours de développement, nouveaux critères à prendre en compte...).

Le programme PhénoFinlait a montré le potentiel de la méthode MIR pour l'analyse à grande échelle des déterminants génétiques et de l'effet des pratiques d'élevage sur la composition du lait et pour le déploiement de nouvelles pistes d'optimisation des processus de production depuis l'élevage jusqu'à la transformation (prévision, différentiation de collecte). La méthode MIR pourrait être mise en œuvre demain pour d'autres objectifs relatifs à la qualité des laits. Il a déjà été mis en évidence que les données spectrales permettent d'estimer la teneur en d'autres composants tels que la lactoferrine (Soyeurt et al 2007), les minéraux (Soyeurt et al 2009), ou même d'évaluer certains critères d'aptitude à la transformation tels que le temps de coagulation ou le raffermissement du caillé (De Marchi et al 2009, Cecchinato et al 2009). Les milliers d'échantillons de lait collectés et conservés à $-80^{\circ} \mathrm{C}$ (Inra CRB-GADIE) ainsi que les bases de données associées (données spectrales, alimentation, génotypage...) sont disponibles pour permettre de nouvelles exploitations du dispositif et de l'investissement PhénoFinlait.

La méthode MIR pourrait aussi être mise en œuvre pour le suivi de la conduite d'élevage. En effet, le lait, dont l'échantillonnage est aisé durant la lactation, et dont la composition résulte à la fois des fermentations dans le rumen, des synthèses mammaires et de transferts directs depuis le sang (urée, protéines sériques...), apparaît de plus en plus comme source de bio-marqueurs ou de signatures permettant d'évaluer le statut physiologique de l'animal et son alimentation. Nous pouvons citer trois travaux d'importance dans ce domaine : $i$ ) la discrimination des laits à l'aide de l'infra-rouge selon leurs conditions de production (race, fourrage dominant, altitude) (Valenti et al 2013); ii) la détection et la valorisation de variations du spectre MIR pour caractériser le statut physiologique (gestation par exemple) et sanitaire (maladies métaboliques, infections mammaires) des femelles dans le cadre de projets tels que le projet européen OptiMir (Dehareng 2011) et les projets labellisés CASDAR AcID et MAMOVICAP ; iii) le développement, pour certaines rations, de modèles prédictifs des rejets de méthane entérique s'appuyant sur le profil en acides gras du lait (14 modèles prédictifs répertoriés par Beillevert 2012).

\section{Conclusion : quelle appropria- tion de ces outils, références et données par les filières ?}

Les produits laitiers sont des produits nobles occupant une place importante dans l'alimentation humaine. Néanmoins les remises en cause (maladies cardiovasculaires et matières grasses animales, intolérances au lactose ou encore allergies) de ces dernières années ainsi que la compétition croissante avec les productions végétales, poussent à mieux valoriser le lait pour ses qualités intrinsèques. En plus du marché alimentaire classique, d'autres voies sont à développer et explorer sur le marché de la nutrition-santé et préventive (Peyraud et Duhem 2013). Pour cela, deux types de travaux sont à conduire parallèlement : i) la caractérisation des bienfaits des constituants du lait et des produits laitiers ; ii) l'établissement d'une série d'outils et références pour le pilotage de la composition du lait depuis la ferme. Le programme PhénoFinlait participe de ce dernier enjeu en se focalisant sur la caractérisation et la maitrise de la composition du lait depuis l'animal jusqu'au tank.

Les outils d'analyse et de prévision de la composition du lait déjà développés et les potentialités de sélection génomique sur ces critères permettront en fonction du choix de la filière d'adapter les différentes étapes du processus de production des produits laitiers depuis l'animal jusqu'à la transformation. Les leviers génétiques et alimentaires considérés conjointement en fonction de leurs potentialités peuvent permettre de produire des laits adaptés à des types de transformation, voire des technologies de transformation différentes. Ce sont là de toutes nouvelles opportunités de pilotage et d'optimisation pour les filières laitières. La question préalable à la mise en œuvre des leviers mis en évidence est l'appropriation de ces outils, et la définition d'objectifs partagés au sein des filières. L'importance et l'efficacité du transfert et de la valorisation des résultats dépendront en particulier des efforts de communication et d'accompagnement des différents acteurs afin de les aider à définir les gains possibles. L'enjeu est de faire fructifier l'investissement scientifique et technique consenti dans le programme PhénoFinlait pour accompagner les éleveurs et leurs filières dans l'amélioration de leur compétitivité à la fois par une optimisation des processus de production actuels, et par l'anticipation sur de nouvelles valorisations (nouveaux produits, nouvelles attentes des consommateurs, nouvelles contraintes de production, protéines à très haute valeur...). Ce 
travail est en cours, il sera long et à géométrie variable selon les bassins de production et les produits. A titre d'exemple, il est vraisemblable que des filières fromagères traditionnelles sous signe de qualité soient parmi les premières à engager des valorisations sur le volet protéique.
Enfin, il convient de préciser que bien que la France ait réalisé là un investissement conséquent et de nature à lui donner des atouts pour l'avenir, plusieurs autres pays se sont lancés dans la « course »: Belgique (Soyeurt et al 2006), Pays-Bas (Visker et al 2006), Danemark et Suède (www.milkgenomics.dk), Italie
(Cecchinato et al 2009), etc. Les autres parties du monde ne sont pas en reste, et la création au milieu des années 2000 de l'International Milk Genomics Consortium (German et al 2006) témoigne du dynamisme sur ces questions scientifiques et techniques.

\section{Références}

Beillevert M., 2012. Evaluation des équations de prédiction du méthane entérique émis par les vaches laitières à partir des caractéristiques de la ration et du profil en acides gras du lait. Rapport de stage. Institut de l'Elevage, Inra, ESA.

Boichard D., Govignon-Gion A., Larroque H., Maroteau C., Palhière I., Tosser-Klopp G., Rupp R., Sanchez M.P., Brochard M., 2014. Déterminisme génétique de la composition en acides gras et protéines du lait des ruminants. In Phénofinlait: Phénotypage et génotypage pour la compréhension et la maîtrise de la composition fine du lait. Brochard M., Boichard D., Brunschwig P., Peyraud J.L. (Eds). Dossier, INRA Prod. Anim., 27, 283-298.

Cecchinato A., De Marchi M., Gallo L., Bittante G., Carnier P., 2009. Mid-infrared spectroscopy predictions as indicator traits in breeding programs for enhanced coagulation properties of milk. J. Dairy Sci., 92, 5304-5313.

Dehareng F., 2011. OptiMIR: new tools for a more sustainable dairy more sustainable dairy sector. ICAR, June 2011, Bourg-en-Bresse, France. http://www.icar.org/Documents/Bourg-enBresse2011/Presentations/session\%204\%20-\% 2023\%20am/1\%20Frederic\%20Dehareng.pdf

De Marchi M., Fagan C.C., O’Donnell C.P., Cecchinato A., Dal Zotto R., Cassandro M., Penasaand M., Bittante G., 2009. Prediction of coagulation properties, titratable acidity, and $\mathrm{pH}$ of bovine milk using mid-infrared spectroscopy. J. Dairy Sci., 92, 423-432.

Ferrand-Calmels M., Palhière I., Brochard M., Leray O., Astruc J. M., Aurel M. R., Barbey S., Bouvier F., Brunschwig P., Caillat H., Douguet M., Faucon-Lahalle F., Gelé M., Thomas G., Trommenschlager J. M., Larroque H., 2014.
Prediction of fatty acid profiles in cow, ewe, and goat milk by mid-infrared spectrometry. J. Dairy Sci., 97, 1-19.

Gelé M., Minery S., Astruc J. M., Brunschwig P., Ferrand M., Lagriffoul G., Larroque H., Legarto J., Leray O., Martin P., Miranda G., Palhière I., Trossat P., Brochard M., 2014. Phénotypage et génotypage à grande échelle de la composition fine des laits dans les filières bovine, ovine et caprine. In Phénofinlait : Phénotypage et génotypage pour la compréhension et la maitrise de la composition fine du lait. Brochard M., Boichard D., Brunschwig P., Peyraud J.L. (Eds). Dossier, INRA Prod. Anim., 27, 255-268.

German J.B., Schanbacher F.L., Lönnerdal B., Medrano J.F., McGuire M.A., McManaman J.L., Rocke D.M., Smith T.P., Neville M.C., Donnelly P., Lange M., Ward R., 2006. International milk genomics consortium. Trends Food Sci. Technol., 17, 656-661.

Legarto L., Gelé M., Ferlay A., Hurtaud C., Lagriffoul G., Palhière I., Peyraud J.L., Rouillé B., Brunschwig P., 2014. Effets des conduites d'élevage sur la production de lait, les taux butyreux et protéique et la composition en acides gras du lait de vache, chèvre et brebis évaluée par spectrométrie dans le moyen infrarouge. In Phénofinlait: Phénotypage et génotypage pour la compréhension et la maîtrise de la composition fine du lait. Brochard M., Boichard D., Brunschwig P., Peyraud J.L. (Eds). Dossier, INRA Prod. Anim., 27, 269282.

Miranda G., Boumahrou N., Bianchi L., Pinard A., Saadaoui B., Guillot A., Henry C., Bevilacqua C., Beauvallet C., Bellier S., Cebo C., Martin P., 2011. Understanding milk protein complexity to produce accurate phenotypes. $8^{\text {th }}$ Int. Milk Genom. Consortium Symp., Melbourne, Australia. Peyraud J.L., Duhem K., 2013. Les élevages laitiers et le lait demain : exercice d'analyse prospective. In : La vache et le lait. Faverdin P., Leroux C., Baumont R. (Eds). Dossier, INRA Prod. Anim., 26, 221-230.

Soyeurt H., Dardenne P., Dehareng F., Lognay G., Veselko G., Marlier M., Bertozzi C., Mayeres P., Gengler N., 2006. Estimating fatty acid content in cow milk using mid-infrared spectrometry. J. Dairy Sci., 89, 3690-3695.

Soyeurt H., Colinet F.G., Arnould V.M.R., Dardenne P., Bertozzi C., Renaville R., Portetelle D., Gengler N., 2007. Genetic variability of lactoferrin content estimated by mid-infrared Spectrometry in bovine milk. J. Dairy Sci., 90, 4443-4450.

Soyeurt H., Bruwier D., Romnee J.M., Gengler N., Bertozzi C., Veselko D., Dardenne P., 2009. Potential estimation of major mineral contents in cow milk using mid-infrared spectrometry. J. Dairy Sci., 92, 2444-2454.

Valenti B., Martin B., Andueza D., Leroux C., Labonne C., Lahalle F., Larroque H., Brunschwig P., Lecomte C., Brochard M., Ferlay A., 2013. Infrared spectroscopic methods for the discrimination of cow milk according to the feeding systems, cow breed and altitude of the dairy farms. Int. Dairy J., 32, 26-32.

Visker M., Heck J., Koks P., Leon-Kloosterziel K., Meuldijk R., Schennink A., Schopen G., Stoop M., Van Arendonk J., 2006. The Dutch Milk Initiative: genetic improvement of milk quality traits and product innovation. $3^{\text {rd }}$ Int. Symp. Milk Genom. Human Health, Brussels, Belgium.

\section{Résumé}

Le programme PhénoFinlait a permis de nombreux développements en matière d'analyse du lait, de mise en relation des facteurs d'élevage et d'alimentation avec la composition fine du lait, et de déterminisme génétique des acides gras et protéines. Cela a été permis, en particulier, par un suivi de quelques 1500 fermes privées. Les filières laitières françaises, bovines, caprines et ovines, disposent, à l'issu de ce programme d'équations d'estimation en routine à partir des données spectrales MIR de la composition fine du lait en acides gras et protéines ; d'une nouvelle méthode hautement résolutive d'analyse qualitative et quantitative des protéines du lait ; de référentiels sur les liens entre les systèmes d'élevage et d'alimentation et la composition fine du lait, allant jusqu'à un outil de prédiction de sa composition en quelques acides gras d'un mois sur l'autre; de populations de référence pour l'évaluation génomique et plus généralement d'une connaissance du déterminisme génétique de ces caractères. Au-delà de ces résultats, le programme PhénoFinlait est également à l'origine de méthodologies relatives à l'exploitation des spectres MIR éprouvées ainsi que de bases de données et de banques d'échantillons (lait et ADN) conséquentes, aisément mobilisables pour aller plus loin dans la caractérisation fine du lait ou pour explorer d'autres domaines (traçabilité des modes de production, suivi des animaux - santé, reproduction -, rejets de méthane entérique...). L'appropriation et la valorisation de ces acquis par les différentes filières est en cours, mais elle est encore très partielle car un travail conséquent d'explication et d'analyse cas par cas des modalités de valorisation les plus adaptées est nécessaire. Par ailleurs, plusieurs nouveaux projets sont issus ou s'appuient en partie sur l'investissement initial PhénoFinlait. Gageons que cela génère encore pendant plusieurs années des connaissances, références et outils nouveaux pour la recherche, les éleveurs et les filières françaises. 


\begin{abstract}
Fine milk composition phenotyping and genotyping: French research and dairy industries invest for the future

Thanks to PhenoFinlait program many knowledge and applications were developed regarding fine milk composition analysis, links between milk composition and farming systems, genetic determinism of milk fatty acids and proteins. Those results are based on a large scale data collection covering about 1,500 commercial farms. French dairy cattle, sheep and goat industries have now access to estimation equations for fatty acids and proteins based on mid infra-red spectra (MIR); very precise qualitative and quantitative analyzing method for milk proteins; references and tools to control milk composition by monitoring dairy females and animal feeding; reference populations for genomic selection and knowledge on genetic determinism of milk fatty acids and proteins profiles. Moreover, thanks to PhenoFinlait program, French research and dairy industries get a huge experience on MIR spectral data; large data-base and sample banks (milk and DNA) available for new developments both to go further on fine milk composition, or to investigate new topics (milk production traceability, animal health and reproduction monitoring, enteric methane emission...). Appropriation and valorization by the different French dairy industries stakeholders is going on, but a lot of work and efforts still has to be done for accompanying field actors in relevant implementation improving competitiveness of their process. Besides, many new research projects come directly from PhenoFinlait program or are partially based on its data, samples and know-how. It is very likely that it will generate new knowledge, references and applications of interest for French researchers, farmers and dairy industries.
\end{abstract}

BROCHARD M., DUHEM K., GESLAIN T., GASTINEL P.-L., PEYRAUD J.-L., 2014. Phénotypage et génotypage de la composition fine du lait : les filières laitières et la recherche française investissent pour l'avenir. In PhénoFinlait: Phénotypage et génotypage pour la compréhension et la maîtrise de la composition fine du lait. Brochard M., Boichard D., Brunschwig P., Peyraud J.-L. (Eds). Dossier, INRA Prod. Anim., 27, 299-302. 